\title{
Communautés rurales et Révolution (1750-1830). Les notables des Mauges
}

\section{Valérie Sottocasa}

\section{(2) OpenEdition \\ 1 Journals}

Édition électronique

URL : https://journals.openedition.org/ahrf/2156

DOI : 10.4000/ahrf.2156

ISSN : 1952-403X

\section{Éditeur :}

Armand Colin, Société des études robespierristes

\section{Édition imprimée}

Date de publication : 1 mars 2005

Pagination : 164-167

ISSN : 0003-4436

\section{Référence électronique}

Valérie Sottocasa, "Communautés rurales et Révolution (1750-1830). Les notables des Mauges»,

Annales historiques de la Révolution française [En ligne], 339 | janvier-mars 2005, mis en ligne le 24 avril 2006, consulté le 24 avril 2022. URL : http://journals.openedition.org/ahrf/2156 ; DOI : https://doi.org/ 10.4000/ahrf.2156

Ce document a été généré automatiquement le 24 avril 2022.

Tous droits réservés 


\title{
Communautés rurales et Révolution (1750-1830). Les notables des Mauges
}

\author{
Valérie Sottocasa
}

\section{RÉFÉRENCE}

Anne Rolland-Boulestreau, Communautés rurales et Révolution (1750-1830). Les notables des Mauges, Rennes, Presses Universitaires de Rennes, 2004, 401 p., ISBN : 2-86847-914-6, 22 e.

1 En choisissant comme angle d'approche la micro-histoire, Anne Rolland-Boulestreau nous propose une plongée dans la vie de trois paroisses des Mauges (Maine-et-Loire) dont la particularité est d'être situées au cœur de la Vendée militaire. L'une d'elle a donné naissance à Jacques Cathelineau, futur chef de la Contre-Révolution vendéenne. L'auteur se propose, à partir de dépouillements très approfondis dans lesquels les papiers notariés occupent une large place, de comprendre comment s'est structurée l'opposition à la Révolution autour des notables des trois paroisses rurales du Pin-enMauges, de Neuvy et de Sainte-Christine, situées entre Cholet et la Loire. Travailler sur un micro-territoire permet de saisir la densité et la richesse des relations sociales, tout particulièrement entre les membres de la notabilité locale, sur laquelle Anne RollandBoulestreau a décidé de centrer son étude. Les liens entre cette élite et les gens du peuple ont donné lieu à nombre d'analyses qui, généralement, mettent l'accent sur l'obéissance populaire dans un cadre quasi immuable depuis le Moyen Âge. Cette image méritait d'être revisitée, tout particulièrement au moment où éclate l'insurrection vendéenne, dont les caractères sociaux et culturels sont éminemment complexes. Cet angle d'approche permet également d'observer les voies selon lesquelles s'est effectuée la politisation dans les communes rurales en rejetant l'idée trop souvent admise de populations maintenues par l'archaïsme à l'écart de toute forme de modernisation culturelle et politique. Enfin, le choix d'un cadre chronologique relativement large 
(1750-1830) permet de poser la question de la place de la crise révolutionnaire dans ces processus.

Composé de trois parties, l'ouvrage s'ouvre sur un tableau de la notabilité locale à la veille de la Révolution, se poursuit par une analyse des réactions des trois communes sélectionnées face à la crise révolutionnaire et s'achève avec la description de l'émergence d'une nouvelle sociabilité forgée à travers l'épreuve de l'insurrection vendéenne. Le livre est abondamment illustré par un jeu de cartes, graphiques et schémas permettant de visualiser la complexité des liens tissés entre les familles de notables et d'en comprendre la nature. L'auteur propose ainsi de synthétiser en quelques graphes leur influence à partir d'actes notariés : un petit nombre d'hommes sont sollicités comme témoins, soit à l'occasion d'emprunts ou de tout autre acte important de la vie quotidienne. Qualifiés «d'honnêtes hommes", ces "notables de référence » reflètent les activités dominantes de leurs communautés : gros métayers à Neuvy ou au Pin, ils sont plutôt issus du monde du commerce dans la paroisse de Sainte-Christine, signe de la symbiose existant entre cette élite locale et les classes populaires. Plus qu'une réalité économique et financière, la qualité de notable est due à la reconnaissance publique.

C'est dans cette perspective que s'inscrit l'étude de la famille de Jacques Cathelineau, à partir de laquelle l'auteur illustre bon nombre de traits de la notabilité rurale dans le micro-territoire choisi. La famille du futur chef contre-révolutionnaire obéit à la plupart des critères déjà repérés par l'auteur : ses membres occupent des fonctions publiques (celle de sacristain est détenue par le père avant d'être occupée par le fils), sont sollicités devant notaire pour leur caution morale et sont souvent choisis comme témoins lors des cérémonies nuptiales dans la paroisse. Ce dernier trait est un signe des liens privilégiés tissés entre familles de notables et de leur intégration dans la paroisse. Un quart des mariages célébrés dans la commune du Pin entre 1752 et 1787 se font en présence de Jacques Cathelineau ou de son père, preuve qu'ils bénéficient d'un réseau de confiance solide. Un autre facteur explique la position de Jacques : sa capacité à réussir sa conversion professionnelle, expression de sa combativité personnelle. Fils de notable, il ne se contente pas de gérer le patrimoine de confiance dont bénéficie sa famille, mais risque une partie de sa fortune pour devenir son propre maître, ce qui lui vaut la reconnaissance publique.

4 Comment cette petite communauté de notables va-t-elle faire face aux bouleversements révolutionnaires ? La vie politique des communes rurales est touchée en profondeur par les réformes entreprises par l'Assemblée nationale. L'observation des comportements électoraux dans les trois paroisses étudiées ne montre aucune homogénéité : quoique voisines, elles suivent des voies différentes, reflet de leur personnalité sociale et économique. Neuvy est dominée par les activités agricoles ; les notables sont exclusivement des métayers, reconduits dans leurs responsabilités publiques lors des premières élections de 1790. Au Pin-en-Mauges, les mêmes hommes occupent des fonctions publiques sous l'Ancien Régime, participent à la rédaction du cahier de doléances et sont élus en 1790 à la tête de la commune. Tout comme à Neuvy, ce sont des hommes du terroir que leur aisance ancienne et leurs savoirs rendent compétents et disponibles au service de leurs concitoyens. Les résultats sont différents pour Sainte-Christine. Tournée vers le commerce, la commune est ouverte sur l'extérieur et compte un grand nombre d'artisans. Plusieurs font leur apparition sur la scène publique à l'occasion des élections de 1790: sur 9 élus, 4 sont des nouveaux 
venus. Au Pin ou à Neuvy, les élus sont portés par toute une parentèle, tout un réseau tissé depuis plusieurs générations. À Sainte-Christine, la Révolution ouvre à des catégories sociales qui en étaient exclues jusqu'alors des charges publiques locales. On pourrait conclure à une politisation plus poussée dans cette dernière commune, ce que l'auteur nuance en affirmant qu'aucune des trois paroisses ne reste en marge des débats et que chacune porte à sa tête les hommes les mieux à même de défendre ses intérêts.

Or la Révolution peut menacer ces intérêts. L'auteur développe l'exemple de la paroisse de Neuvy dont l'église est susceptible d'être fermée du fait des regroupements de paroisses. Cette perspective inquiète les habitants, car ce serait là une atteinte à l'identité communautaire. Les notables traditionnels semblent les plus qualifiés pour défendre une identité dont ils sont partie prenante depuis plusieurs générations. Au Pin, les cahiers de doléances attestent une volonté de changement qui vient se briser sur les réformes religieuses perçues comme une menace et une source d'insécurité pour la collectivité : tout comme à Neuvy, la commune se ferme face à la Révolution. Les marchands et artisans de Sainte-Christine voient au contraire dans les réformes révolutionnaires l'occasion de gagner en puissance à l'échelle locale, leur commune espérant devenir chef-lieu de canton. La religion n'est pas la seule source de déstabilisation des sociétés rurales: la vente des biens nationaux représente un important transfert foncier qui peut remettre en question les équilibres économiques et sociaux traditionnels. Dans les communes de Neuvy et du Pin, les notables locaux n'achètent pas ou peu de biens vendus à cette occasion ; à Sainte-Christine, ils achètent modestement ce type de terres.

Lors des élections suivantes, qui se déroulent à la fin de 1792, le comportement électoral du groupe des notables et des électeurs témoigne de changements sensibles. Peu d'élus se représentent. Désavouant l'évolution politique, ils adoptent une sorte de réserve et sont remplacés par des notables plus modestes. Il ne faut pas y voir un vote sanction: les nouveaux élus appartiennent aux mêmes réseaux et parentèles que les sortants. Les notables sont en fait confirmés dans leur choix vis-à-vis de la Révolution ; leur attitude traduit les réticences de la communauté. Ceux qui s'engagent plus tard dans la révolte vendéenne le font donc avec un capital de confiance non négligeable. L'auteur se penche sur l'exemple de Jacques Cathelineau. Au printemps 1793, alors que gronde la colère des habitants des campagnes contre les levées d'hommes, Cathelineau décide de rejoindre les premiers insurgés. Vingt-sept hommes originaires du Pin se joignent à lui ; leurs noms sont connus grâce à deux listes fournies par l'abbé réfractaire de la paroisse, camarade du chef rebelle, et par le fils de l'un des premiers compagnons de Cathelineau. Relativement peu nombreux, ces hommes ont néanmoins joué un rôle clé dans le déclenchement de la révolte. La moitié d'entre eux sont des célibataires en âge de participer à la levée ; un tiers est d'origine paysanne, les deux tiers sont des artisans. Contrairement aux idées reçues, la composition de la troupe de Cathelineau prouve que l'insurrection n'est pas essentiellement paysanne. D'autres études montrent que la part des non natifs est loin d'être aussi négligeable au sein des compagnies paroissiales que ce qu'on a pu écrire. En revanche, les liens familiaux y tiennent une place importante, ce qui assure une forte cohésion interne sans exclure des combattants extérieurs à la commune.

7 La guerre met en évidence des engagements antagonistes : les " patriotes » vendéens, qui se recrutent essentiellement à Sainte-Christine, sont pour la plupart des artisans 
modestes et récemment implantés dans la paroisse : ils ne sont pas intégrés dans des réseaux de parenté solides et développés. Du côté des «blancs ", des comités royalistes s'organisent très tôt. Il n'y a donc pas d'unanimité dans l'engagement vendéen. C'est pourquoi la reconstruction du pays se révèle particulièrement difficile. La mémoire populaire reste longtemps marquée par le souvenir des violences contre les populations civiles. Le retour de la confiance ne peut se faire en dehors des notables qui, paradoxalement, se sont mis au service de la rébellion, mais restent les artisans incontournables de la pacification. Les aléas de la politique thermidorienne puis directoriale ne favorisent pas le rétablissement de cette confiance alors même que les différents gouvernements privilégient les propriétaires terriens comme intermédiaires sociaux. D'importantes modifications interviennent dans la composition des élites locales: à Sainte-Christine, les commerçants traditionnellement dominants sont remplacés par des hommes de la terre; partout, les nobles investissent les postes de commandement qu'ils délaissaient sous l'Ancien Régime. Ces derniers sont enracinés dans le village : les réseaux familiaux se consolident. La participation à l'insurrection vendéenne est désormais une condition nécessaire pour obtenir la confiance des populations locales. Cela vaut aussi pour la noblesse dont la participation à l'insurrection a été relativement peu importante, les uns ayant opté pour l'émigration, les autres pour la clandestinité, la propriété étant confiée aux femmes afin d'éviter sa vente au titre de bien national. Quoi qu'il en soit, ces familles ont souffert sous la Révolution et ont partagé avec les populations des épreuves communes. La nouvelle place de la noblesse au sein des communes est légitimée par la guerre de Vendée.

Ce passé commun est recomposé à des fins d'identité. L'Empire reconnaît la Vendée pour mieux la surveiller, alors que sous la Restauration, les combattants sont valorisés pour leur engagement passé. Les nombreux dossiers de demande de pension constitués entre 1814 et 1824 permettent de mieux connaître les Vendéens, mais aussi les liens de solidarité entretenus au moment de l'insurrection et dans les années suivantes. Les solliciteurs, en effet, doivent trouver des témoins pour attester leur engagement et témoigner de leurs actes de bravoure. Vingt ou trente ans plus tard, les solidarités nouées pendant la guerre se reconstituent, ce qui participe d'un travail de mémoire en cours d'élaboration. Vers 1830, il devient possible de mesurer les évolutions qui ont affecté la société locale. Les pratiques matrimoniales en sont un bon révélateur; elles permettent de constater que Sainte-Christine se replie sur elle-même au lendemain de la Révolution, tant du fait des destructions matérielles que des divisions politiques. À Neuvy et au Pin, la part des amis parmi les témoins a augmenté et traduit l'importance des liens tissés au cours des guerres de Vendée. Au Pin, les compagnons de Jacques Cathelineau sont très sollicités ; plusieurs mariages sont conclus entre leurs familles. Le témoin n'est plus seulement un membre reconnu de la communauté des notables locaux, mais un combattant honoré par la collectivité.

Le choix de la micro-histoire se révèle donc riche en enseignements. Il permet d'introduire d'importantes nuances dans l'image de la Vendée rebelle. Le rôle de la petite notabilité a été à la fois conforté et valorisé par les guerres qui deviennent un prisme à travers lequel se décrypte la sociabilité au lendemain de la Révolution. Elle a gagné en autorité là où elle aurait pu être déstabilisée, car elle a pu compter sur un enracinement et des liens de confiance anciens, dans le cadre de réseaux de parenté et d'amitié que les archives notariales révèlent dans toute leur complexité. Devenus un intermédiaire incontournable entre la communauté et l'État, les petits notables rejoints par la noblesse locale se sont imposés en participant activement à la reconstruction 
d'un pays traumatisé et à l'élaboration d'une mémoire créatrice d'identité. La microhistoire permet de pénétrer les liens sociaux, d'en comprendre les détails et d'en rendre l'infinie richesse. Le cadre étroit du territoire de la recherche peut alors donner naissance à une analyse fine, novatrice et enrichissante sur une région saturée d'histoire. 